International Journal of Pure and Applied Mathematics

Volume 99 No. 1 2015, 65-76

ISSN: 1311-8080 (printed version); ISSN: 1314-3395 (on-line version)

url: http://www.ijpam.eu

doi: http://dx.doi.org/10.12732/ijpam.v99i1.6

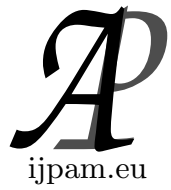

\title{
ON FUZZY INVENTORY MODEL WITH ALLOWABLE SHORTAGE
}

\author{
D. Stephen Dinagar ${ }^{1}$, J. Rajesh Kannan ${ }^{2}$ \\ ${ }^{1}$ PG. and Research Department of Mathematics \\ T.B.M.L College \\ Porayar, INDIA \\ ${ }^{2}$ Department of Basic Engineering \\ S.S.P. College \\ Puthur, INDIA
}

\begin{abstract}
In this paper, the fuzzy inventory model with allowable shortage has been considered in a fuzzy environment using the new hexagonal fuzzy numbers. Our goal is to determine the fuzzy optimal total cost and fuzzy optimal order quantity for the proposed inventory model. Holding cost, Ordering cost, Shortage cost and Demand are taken as in terms of Hexagonal fuzzy numbers. New arithmetic operations are defined and applied in sensitivity analysis. A relevant numerical example is also included, to justify the proposed notion.
\end{abstract}

AMS Subject Classification: 03E72, 90B05

Key Words: hexagonal fuzzy numbers, fuzzy inventory model, fuzzy optimal total cost, fuzzy optimal order quantity, allowable shortage

\section{Introduction}

Inventory model was developed by Harris [3], Wilson [9] aroused interest in the EOQ model in academics and industries. In 1970, Zadeh et al. [11] proposed some strategies for decision making in fuzzy environment. Jain [4] worked on decision making in the presence of fuzzy decision making models. Thus, EOQ

Received: July 31, 2014

(c) 2015 Academic Publications, Ltd. url: www.acadpubl.eu 
model serves a useful approximation to many real life problems. Urgeletti [8] treated EOQ model in fuzzy sense, and used triangular fuzzy number. Chen and Wang [1] used trapezoidal fuzzy number to fuzzify the order cost, inventory cost, and back order cost in the total cost of inventory model without backorder. Further, in a series of papers, Yao et al. [10], considered the fuzzified problems for the inventory with or without backorder models. Kacpryzk et al. [5] discussed some long-term inventory policy making through the fuzzydecision making models. Later, Hadley et al [2] analyzed many inventory systems. Parvathi and Gajalakshmi [6] investigate a deterministic inventory model with allowable shortage.

In this paper, a new fuzzy number called 'Hexagonal fuzzy numbers' is utilized in developing the notion of Inventory model. Though Rajarajesvari et.al [7] proposed the above said fuzzy number without any restrictions of parameter , We have modified the definition of the number by including conditions for the convexity of the number and few more results are also included in the work. The main aim of the authors is to estimate the fuzzy optimal order quantity and fuzzy optimal total cost of an inventory system under study due to irregularities or physical properties of the parameter variables. For this situation we apply fuzzy concepts, shortage is allowed and it is completely backlogged. An algorithm is developed to find the fuzzy total cost. Sensitivity analysis is carried out through the numerical examples.

In this article, in Section 2, some basic definitions and arithmetic operations on hexagonal fuzzy numbers are presented. In Section 3, we describe in brief the notions and assumptions used in the developed Fuzzy inventory model Formulation and Analysis of the inventory model in fuzzy sense and algorithm are presented. In Section 4, a numerical example is given to illustrate the model and sensitivity analysis has been made for different changes in the parameter values. In Section 5, the concluding remarks are also given.

\section{Definitions and Preliminaries}

Definition 2.1 (Fuzzy Set). A Fuzzy set $\tilde{A}$ in a universe of discourse $x$ is defined as the following set of pairs $\tilde{A}=\left\{\left(x, \mu_{\tilde{A}}(x)\right): x \in X\right\}$. Here $\mu_{\tilde{A}}: X \rightarrow[0,1]$ is a mapping called the membership value of $x \in X$ in a fuzzy set $\tilde{A}$.

Definition 2.2 (Convex Fuzzy Set). A fuzzy set $\tilde{A}=\left\{\left(x, \mu_{\tilde{A}}(x)\right)\right\} \subseteq X$ is called convex fuzzy set if all $\tilde{A}_{x}$ are convex sets i.e. for every element $x_{1} \in A_{\alpha}$ and $x_{2} \in A_{\alpha}$ for every $\alpha \in[0,1] \lambda x_{1}+(1-\lambda) x_{2} \in A_{\alpha} \forall \lambda \in[0,1]$. Otherwise the 
fuzzy set is called non convex fuzzy set.

Definition 2.3 (Hexagonal Fuzzy Number). A fuzzy number on $\tilde{A}_{h}$ is a Hexagonal fuzzy number denoted by $\tilde{A}_{h}=\left(a_{1}, a_{2}, a_{3}, a_{4}, a_{5}, a_{6}\right)$ where $\left(a_{1} \leq\right.$ $\left.a_{2} \leq a_{3} \leq a_{4} \leq a_{5} \leq a_{6}\right)$ are real numbers Satisfying $a_{2}-a_{1} \leq a_{3}-a_{2}$ and $a_{5}-a_{4} \geq a_{6}-a_{5}$ and its membership function $\mu_{\tilde{A}_{h}}(x)$ is given as;

$$
\mu_{\tilde{A}_{h}}(x)= \begin{cases}0, & x<a_{1} \\ \frac{1}{2}\left(\frac{x-a_{1}}{a_{2}-a_{1}}\right), & a_{1} \leq x \leq a_{2} \\ \frac{1}{2}+\frac{1}{2}\left(\frac{x-a_{2}}{a_{3}-a_{2}}\right), & a_{2} \leq x \leq a_{3} \\ 1, & a_{3} \leq x \leq a_{4} \\ 1-\frac{1}{2}\left(\frac{x-a_{4}}{a_{5}-a_{4}}\right), & a_{4} \leq x \leq a_{5} \\ \frac{1}{2}\left(\frac{a_{6}-x}{a_{6}-a_{5}}\right), & a_{5} \leq x \leq a_{6} \\ 0, & x>a_{6} .\end{cases}
$$

\section{Remark 2.4.}

(1) The Hexagonal fuzzy numbers $\tilde{A}_{h}$ becomes trapezoidal fuzzy numbers if $a_{2}-a_{1}=a_{3}-a_{2}$ and $a_{5}-a_{4}=a_{6}-a_{5}$

(2) The Hexagonal fuzzy numbers $\tilde{A}_{h}$ becomes non-convex fuzzy numbers if $a_{2}-a_{1}>a_{3}-a_{2}$ and $a_{5}-a_{4}<a_{6}-a_{5}$

Definition 2.5 (Equality of Two Hexagonal Fuzzy Numbers). Two Hexagonal fuzzy numbers $\tilde{A}=\left(a_{1}, a_{2}, a_{3}, a_{4}, a_{5}, a_{6}\right)$ and $\tilde{B}=\left(b_{1}, b_{2}, b_{3}, b_{4}, b_{5}, b_{6}\right)$ are said to be equal i.e. $\tilde{A}=\tilde{B}$ if and only if $a_{1}=b_{1}, a_{2}=b_{2}, a_{3}=b_{3}, a_{4}=b_{4}, a_{5}=$ $b_{5}, a_{6}=b_{6}$.

Definition 2.6 (Symmetric Hexagonal fuzzy number). A fuzzy number $\tilde{A}=\left(a_{1}, a_{2}, a_{3}, a_{4}, a_{5}, a_{6}\right)$ is said to be a symmetric Hexagonal fuzzy number, if $a_{3}-a_{1}=a_{6}-a_{4}$. Otherwise the fuzzy number is called Non symmetric fuzzy number.

Definition 2.7. A Hexagonal fuzzy number $\tilde{A}=\left(a_{1}, a_{2}, a_{3}, a_{4}, a_{5}, a_{6}\right)$ is said to be none negative (non positive) i.e. $\tilde{A} \geq 0(\tilde{A} \leq 0)$ if and only if $a_{1} \geq 0\left(a_{6} \leq 0\right)$.

Definition 2.8 (New Arithmetic Operations). The new arithmetic operations between hexagonal fuzzy numbers proposed are given below. Let us consider $\tilde{A}_{1}=\left(a_{1}, a_{2}, a_{3}, a_{4}, a_{5}, a_{6}\right)$ and $\tilde{A}_{2}=\left(b_{1}, b_{2}, b_{3}, b_{4}, b_{5}, b_{6}\right)$ be two hexagonal fuzzy numbers. Then, 
Table 1: Different types of hexagonal fuzzy numbers.

\begin{tabular}{l} 
Types of Hexagonal \\
fuzzy number \\
$\tilde{A}=\left(a_{1}, a_{2}, a_{3}, a_{4}, a_{5}, a_{6}\right)$ \\
\hline \\
Hexagonal fuzzy numbers \\
$\tilde{A}_{h}$ becomes trapezoidal \\
fuzzy numbers
\end{tabular}

Convex Hexagonal fuzzy numbers $\tilde{A}_{h}$

Conditions

Pictorial representations

Non convex Hexagonal fuzzy numbers $\tilde{A}_{h}$
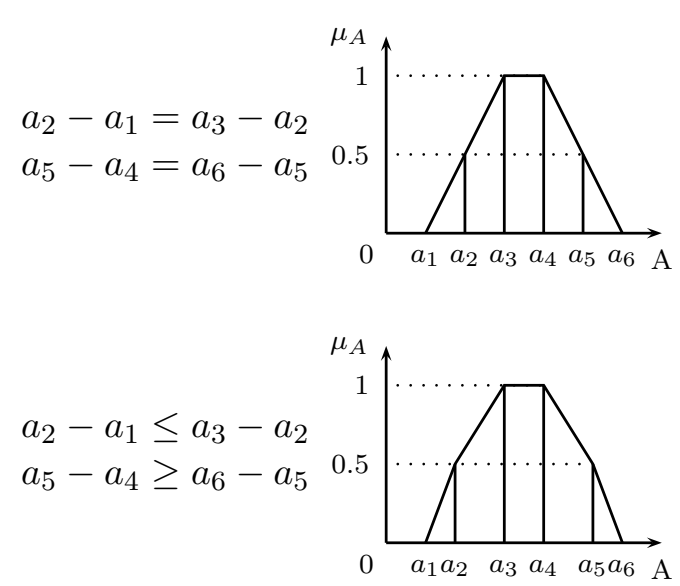

Symmetric Hexagonal fuzzy numbers $\tilde{A}_{h}$
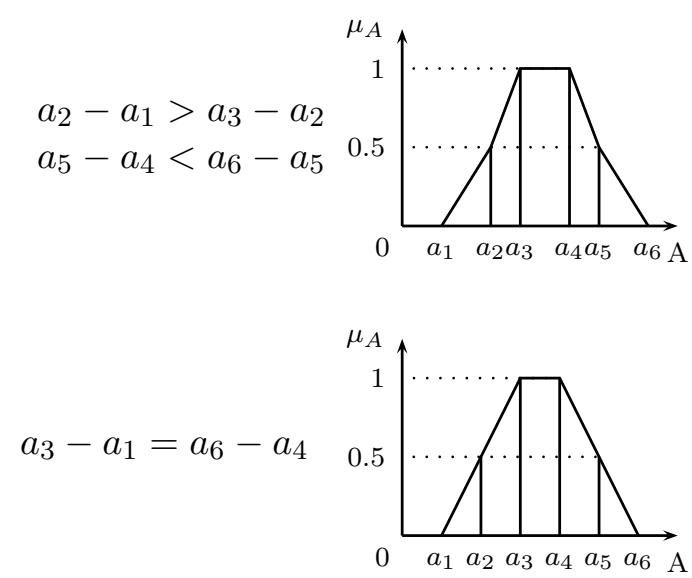

(i) The addition of $\tilde{A}_{1}$ and $\tilde{A}_{2}$ is

$$
\tilde{A}_{1}(+) \tilde{A}_{2}=\left(a_{1}+b_{1}, a_{2}+b_{2}, a_{3}+b_{3}, a_{4}+b_{4}, a_{5}+b_{5}, a_{6}+b_{6}\right) \text {. }
$$

(ii) The subtraction of $\tilde{A}_{1}$ and $\tilde{A}_{2}$ is

$$
\tilde{A}_{1}(-) \tilde{A}_{2}=\left(a_{1}-b_{6}, a_{2}-b_{5}, a_{3}-b_{4}, a_{4}-b_{3}, a_{5}-b_{2}, a_{6}-b_{1}\right) \text {. }
$$

(iii) The multiplication of $\tilde{A}_{1}$ and $\tilde{A}_{2}$ is 
Table 1: Continuation: Different types of hexagonal fuzzy numbers.

Non symmetric Hexagonal fuzzy numbers type-1 $\left(l_{s}>r_{s}\right)$ $a_{3}-a_{1}>a_{6}-a_{4}$

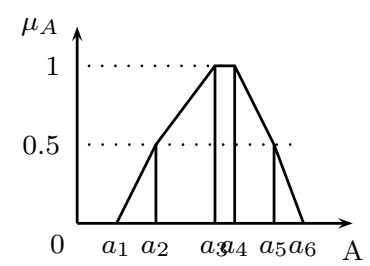

Non symmetric Hexagonal fuzzy numbers type-2 $\left(l_{s}<r_{s}\right)$ $a_{3}-a_{1}<a_{6}-a_{4}$

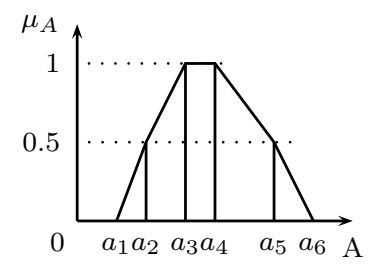

Perfect symmetric

Hexagonal fuzzy numbers

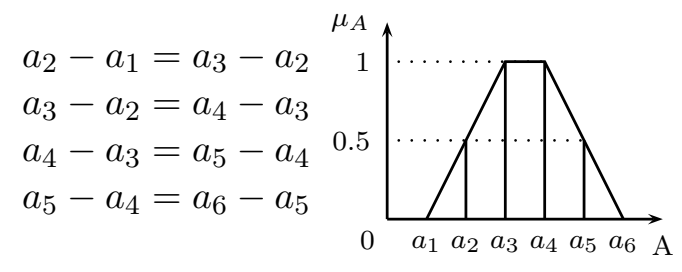

$\tilde{A}_{1}(\times) \tilde{A}_{2}=\left(\frac{a_{1}}{6} \sigma_{b}, \frac{a_{2}}{6} \sigma_{b}, \frac{a_{3}}{6} \sigma_{b}, \frac{a_{4}}{6} \sigma_{b}, \frac{a_{5}}{6} \sigma_{b}, \frac{a_{6}}{6} \sigma_{b}\right)$,

where $\sigma_{b}=\left(b_{1}+b_{2}+b_{3}+b_{4}+b_{5}+b_{6}\right)$.

(iv) The division of $\tilde{A}_{1}$ and $\tilde{A}_{2}$ is

$\tilde{A}_{1}(\div) \tilde{A}_{2}=\left(\frac{6 a_{1}}{\sigma_{b}}, \frac{6 a_{2}}{\sigma_{b}}, \frac{6 a_{3}}{\sigma_{b}}, \frac{6 a_{4}}{\sigma_{b}}, \frac{6 a_{5}}{\sigma_{b}}, \frac{6 a_{6}}{\sigma_{b}}\right)$

if $\sigma_{b} \neq 0$ where $\sigma_{b}=\left(b_{1}+b_{2}+b_{3}+b_{4}+b_{5}+b_{6}\right)$.

(v) If $k \neq 0$ is a scalar $k \tilde{A}$ is defined as

$k \tilde{A}= \begin{cases}\left(k a_{1}, k a_{2}, k a_{3}, k a_{4}, k a_{5}, k a_{6}\right), & \text { if } k>0 \\ \left(k a_{6}, k a_{5}, k a_{4}, k a_{3}, k a_{2}, k a_{1}\right), & \text { if } k<0\end{cases}$

(vi) $\sqrt{\tilde{A}}=\sqrt{a_{1}, a_{2}, a_{3}, a_{4}, a_{5}, a_{6}}=\left(\sqrt{a_{1}}, \sqrt{a_{2}}, \sqrt{a_{3}}, \sqrt{a_{4}}, \sqrt{a_{5}}, \sqrt{a_{6}}\right)$. where $a_{1}, a_{2}, a_{3}, a_{4}, a_{5}, a_{6}$ are non zero positive real numbers.

Definition 2.9. We define a ranking function $R: f(R) \rightarrow R$ which maps each fuzzy numbers to the real line: $f(R)$ represents the set of all hexagonal 
fuzzy numbers. If $R$ be any linear ranking function, then $R(\tilde{A})=\left(\frac{a_{1}+a_{2}+a_{3}+a_{4}+a_{5}+a_{6}}{6}\right)$.

Definition 2.10 (Equivalent Hexagonal Fuzzy Numbers). A fuzzy number $\tilde{A}$ is said to be equivalent to a fuzzy number $\tilde{B}$ if its value of the Ranking function are the same. i.e. $\tilde{A} \approx \tilde{B}$ if $R(\tilde{A})=R(\tilde{B})$.

\section{Fuzzy Inventory Model}

\subsection{Notations}

We define the following symbols:

$\tilde{C}_{h}$ : $\quad$ Fuzzy holding cost per unit quantity per unit time

$\tilde{C}_{a}$ : $\quad$ Fuzzy Setup cost (or) ordering cost per order

$T$ : Length of the plan

$\tilde{C}_{s}: \quad$ Fuzzy Shortage cost per unit quantity

$\tilde{D}_{t}: \quad$ Fuzzy Total demand over the planning time period $[0, T]$

$\tilde{Q}: \quad \quad$ Fuzzy Order quantity per cycle

$\tilde{T}_{c}$ : $\quad$ Fuzzy total cost for the period $[0, T]$

$\tilde{F}(Q)^{*}: \quad$ Minimum Fuzzy total cost for $[0, T]$

$\tilde{Q}_{d}^{*}: \quad$ Fuzzy Optimal order quantity

\subsection{Assumptions}

In the present paper, the assumptions considered are as follows:

(i) Shortage cost is fuzzy nature

(ii) Total demand is fuzzy nature

(iii) Time plan is constant

(iv) Holding cost, Ordering cost are fuzzy in nature 


\subsection{Formulation and Analysis of the Model}

We consider the model with allowable shortage in fuzzy environment since the fuzzy total demand with time period $[0, T]$, fuzzy ordering cost per order fuzzy carrying cost (or) holding cost per unit quantity per unit time and fuzzy shortage cost per unit quantity are in fuzzy nature. Our goal is to determine the fuzzy optimal total cost and fuzzy optimal order quantity for the proposed fuzzy inventory model. Now we fuzzifying total cost is given by

$$
\tilde{T}_{c}=\frac{\tilde{H} T \tilde{Q} \tilde{S}^{2}}{2(\tilde{H} T+\tilde{S})^{2}}+\frac{\tilde{S} \tilde{Q}\left(1-\frac{\tilde{S}}{\tilde{H} T+\tilde{S}}\right)^{2}}{2}+\frac{\tilde{A} \tilde{D}}{\tilde{Q}} .
$$

Our aim is to obtain fuzzy total cost and the optimal order quantity in terms of hexagonal fuzzy numbers by using simple calculus techniques. If $\tilde{C}_{h}, \tilde{C}_{a}, \tilde{C}_{s}$, $\tilde{D}_{t}$ denote the hexagonal fuzzy numbers defined by

$$
\begin{aligned}
& \tilde{C}_{h}=\left(C_{h_{1}}, C_{h_{2}}, C_{h_{3}}, C_{h_{4}}, C_{h_{5}}, C_{h_{6}}\right) \\
& \tilde{D}_{t}=\left(D_{t_{1}}, D_{t_{2}}, D_{t_{3}}, D_{t_{4}}, D_{t_{5}}, D_{t_{6}}\right) \\
& \tilde{C}_{a}=\left(C_{a_{1}}, C_{a_{2}}, C_{a_{3}}, C_{a_{4}}, C_{a_{5}}, C_{a_{6}}\right) \\
& \tilde{C}_{s}=\left(C_{s_{1}}, C_{s_{2}}, C_{s_{3}}, C_{s_{4}}, C_{s_{5}}, C_{s_{6}}\right) \\
& \tilde{T}_{c}=\left\{\frac{\tilde{C}_{h} T \tilde{Q}\left(\tilde{C}_{s}\right)^{2}}{2\left(\tilde{C}_{h} T+\tilde{C}_{s}\right)^{2}}+\frac{\tilde{C}_{s}\left(1-\frac{\tilde{C}_{s}}{\tilde{C}_{h} T+\tilde{C}_{s}}\right)^{2}}{2}+\frac{\tilde{C}_{a} \tilde{D}_{t}}{\tilde{Q}}\right\} .
\end{aligned}
$$

By using new arithmetic operations and simplifying we get 


$$
\begin{aligned}
& \tilde{T}_{C}=\left\{\begin{array}{l}
\frac{\frac{T \tilde{Q}}{6} C_{s_{1}} \partial_{a} \partial_{b}}{\partial_{c}}+\frac{\tilde{Q}}{12}\left(\frac{\left(T C_{h_{1}}+C_{s_{1}}-C_{s_{6}}\right) \partial_{a}}{\partial_{d}} \partial_{e}\right)+\frac{1}{6 \tilde{Q}} D_{t_{1}} \partial_{f}, \\
\frac{\frac{T \tilde{Q}}{6} C_{s_{2}} \partial_{a} \partial_{b}}{\partial_{c}}+\frac{\tilde{Q}}{12}\left(\frac{\left(T C_{h_{2}}+C_{s_{2}}-C_{s_{5}}\right) \partial_{a}}{\partial_{d}} \partial_{e}\right)+\frac{1}{6 \tilde{Q}} D_{t_{2}} \partial_{f}, \\
\frac{\frac{T \tilde{Q}}{6} C_{s_{3}} \partial_{a} \partial_{b}}{\partial_{c}}+\frac{\tilde{Q}}{12}\left(\frac{\left(T C_{h_{3}}+C_{s_{3}}-C_{s_{4}}\right) \partial_{a}}{\partial_{d}} \partial_{e}\right)+\frac{1}{6 \tilde{Q}} D_{t_{3}} \partial_{f}, \\
\frac{\frac{T \tilde{Q}}{6} C_{s_{4}} \partial_{a} \partial_{b}}{\partial_{c}}+\frac{\tilde{Q}}{12}\left(\frac{\left(T C_{h_{4}}+C_{s_{4}}-C_{s_{3}}\right) \partial_{a}}{\partial_{d}} \partial_{e}\right)+\frac{1}{6 \tilde{Q}} D_{t_{4}} \partial_{f}, \\
\frac{\frac{T \tilde{Q}}{6} C_{s_{5}} \partial_{a} \partial_{b}}{\partial_{c}}+\frac{\tilde{Q}}{12}\left(\frac{\left(T C_{h_{5}}+C_{s_{5}}-C_{s_{2}}\right) \partial_{a}}{\partial_{d}} \partial_{e}\right)+\frac{1}{6 \tilde{Q}} D_{t_{5}} \partial_{f}, \\
\frac{\frac{T \tilde{Q}}{6} C_{s_{6}} \partial_{a} \partial_{b}}{\partial_{c}}+\frac{\tilde{Q}}{12}\left(\frac{\left(T C_{h_{6}}+C_{s_{6}}-C_{s_{1}}\right) \partial_{a}}{\partial_{d}} \partial_{e}\right)+\frac{1}{6 \tilde{Q}} D_{t_{6}} \partial_{f}
\end{array}\right. \\
& \tilde{T}_{c}=\left(a_{1}, a_{2}, a_{3}, a_{4}, a_{5}, a_{6}\right)=F(\tilde{Q})
\end{aligned}
$$

where

$$
\begin{aligned}
\partial_{a} & =\left(C_{s_{1}}+C_{s_{1}}+C_{s_{1}}+C_{s_{1}}+C_{s_{1}}+C_{s_{6}}\right), \\
\partial_{b} & =\left(C_{h_{1}}+C_{h_{2}}+C_{h_{3}}+C_{h_{4}}+C_{h_{5}}+C_{h_{6}}\right) \\
\partial_{c} & =\left[\frac{T C_{h_{1}}+C_{s_{1}}}{3}\left(T C_{h_{1}}+C_{s_{1}}+\cdots+T C_{h_{6}}+C_{s_{6}}\right)\right. \\
& \left.+\cdots+\frac{T C_{h_{6}}+C_{s_{6}}}{3}\left(T C_{h_{1}}+C_{s_{1}}+\cdots+T C_{h_{6}}+C_{s_{6}}\right)\right] \\
\partial_{d} & =\left(T C_{h_{1}}+C_{s_{1}}+\cdots+T C_{h_{6}}+C_{s_{6}}\right), \\
\partial_{e}= & \left(\frac{\left(6 T C_{h_{1}}+C_{s_{1}}-C_{s_{6}}\right)}{\left(T C_{h_{1}}+C_{s_{1}}+\cdots+T C_{h_{6}}+C_{s_{6}}\right)}\right. \\
& \left.+\cdots+\frac{\left(6 T C_{h_{6}}+C_{s_{6}}-C_{s_{1}}\right)}{\left(T C_{h_{1}}+C_{s_{1}}+\cdots+T C_{h_{6}}+C_{s_{6}}\right)}\right) \\
\partial_{f}= & \left(C_{a_{1}}+C_{a_{2}}+C_{a_{3}}+C_{a_{4}}+C_{a_{5}}+C_{a_{6}}\right), \\
\partial_{g}= & \left(Q_{d 1}+Q_{d 2}+Q_{d 3}+Q_{d 4}+Q_{d 5}+Q_{d 6}\right) .
\end{aligned}
$$

The fuzzy optimal order quantity $\tilde{Q}_{d}^{*}$ which is minimize the total inventory $\operatorname{cost} \tilde{T}_{c}=F(\tilde{Q})$ is obtained as the solution of the first order fuzzy differential 
equation $\frac{d}{d \tilde{Q}}\left(\tilde{T}_{c}\right)=\tilde{0}$ and it is found as

$$
\tilde{Q}_{d}^{*}=\sqrt{\begin{array}{l}
\frac{2 D_{t_{1}} \partial_{f}}{\partial_{a}}+\frac{D_{t_{1}} \partial_{f}}{T \partial_{b}}, \frac{2 D_{t_{2}} \partial_{f}}{\partial_{a}}+\frac{D_{t_{2}} \partial_{f}}{T \partial_{b}}, \frac{2 D_{t_{3}} \partial_{f}}{\partial_{a}}+\frac{D_{t_{3}} \partial_{f}}{T \partial_{b}} \\
\frac{2 D_{4} \partial_{f}}{\partial_{a}}+\frac{D_{t_{4}} \partial_{f}}{T \partial_{b}}, \frac{2 D_{t_{5}} \partial_{f}}{\partial_{a}}+\frac{D_{t_{5}} \partial_{f}}{T \partial_{b}}, \frac{2 D_{t_{6}} \partial_{f}}{\partial_{a}}+\frac{D_{t_{6}} \partial_{f}}{T \partial_{b}}
\end{array}} .
$$

$\tilde{Q}_{d}^{*}=\left(Q_{d 1}, Q_{d 2}, Q_{d 3}, Q_{d 4}, Q_{d 5}, Q_{d 6}\right)$ by using Definition 2.8(vi).

Also $\tilde{Q}=\tilde{Q}_{d}^{*}$ we have $\frac{d^{2} F(\tilde{Q})}{d \tilde{Q}^{2}}>0$.

This shows that $F(\tilde{Q})$ is minimum at $\tilde{Q}=\tilde{Q}_{d}^{*}$ and from (1).

$$
F\left(Q^{*}\right)=\left\{\begin{array}{l}
\frac{\frac{T}{36} C_{s_{1}} \partial_{a} \partial_{b} \partial_{g}}{\partial_{c}}+\frac{1}{72}\left(\frac{\left(T C_{h_{1}}+C_{s_{1}}-C_{s_{6}}\right) \partial_{a} \partial_{g}}{\partial_{d}} \partial_{e}\right)+\frac{D_{t_{1}} \partial_{f}}{\partial_{g}} \\
\frac{\frac{T}{36} C_{s_{2}} \partial_{a} \partial_{b} \partial_{g}}{\partial_{c}}+\frac{1}{72}\left(\frac{\left(T C_{h_{2}}+C_{s_{2}}-C_{s_{5}}\right) \partial_{a} \partial_{g}}{\partial_{d}} \partial_{e}\right)+\frac{D_{t_{2}} \partial_{f}}{\partial_{g}} \\
\frac{\frac{T}{36} C_{s_{3}} \partial_{a} \partial_{b} \partial_{g}}{\partial_{c}}+\frac{1}{72}\left(\frac{\left(T C_{h_{3}}+C_{s_{3}}-C_{s_{4}}\right) \partial_{a} \partial_{g}}{\partial_{d}} \partial_{e}\right)+\frac{D_{t_{3}} \partial_{f}}{\partial_{g}} \\
\frac{\frac{T}{36} C_{s_{4}} \partial_{a} \partial_{b} \partial_{g}}{\partial_{c}}+\frac{1}{72}\left(\frac{\left(T C_{h_{4}}+C_{s_{4}}-C_{s_{3}}\right) \partial_{a} \partial_{g}}{\partial_{d}} \partial_{e}\right)+\frac{D_{t_{4}} \partial_{f}}{\partial_{g}} \\
\frac{\frac{T}{36} C_{s_{5}} \partial_{a} \partial_{b} \partial_{g}}{\partial_{c}}+\frac{1}{72}\left(\frac{\left(T C_{h_{5}}+C_{s_{5}}-C_{s_{2}}\right) \partial_{a} \partial_{g}}{\partial_{d}} \partial_{e}\right)+\frac{D_{t_{5}} \partial_{f}}{\partial_{g}} \\
\frac{\frac{T}{36} C_{s_{6}} \partial_{a} \partial_{b} \partial_{g}}{\partial_{c}}+\frac{1}{72}\left(\frac{\left(T C_{h_{6}}+C_{s_{6}}-C_{s_{1}}\right) \partial_{a} \partial_{g}}{\partial_{d}} \partial_{e}\right)+\frac{D_{t_{6}} \partial_{f}}{\partial_{g}}
\end{array}\right.
$$

\subsection{Algorithm for Finding Fuzzy Optimal Total Cost and Fuzzy Optimal Order Quantity}

\section{Step 1:}

Calculate the model fuzzy total cost for the fuzzy values of $\tilde{C}_{h}, \tilde{C}_{a}, \tilde{C}_{s}, \tilde{D}_{t}$.

Step 2:

Now determine fuzzy total cost using new arithmetic operations fuzzy holding cost, fuzzy ordering cost, fuzzy shortage cost and fuzzy demand taken in terms of hexagonal fuzzy numbers

\section{Step 3:}

Find the fuzzy optimal order quantity which can be obtain by putting the first derivative of $F(\tilde{Q})$ equal to zero and second derivate is positive at $\tilde{Q}=\tilde{Q}_{d}^{*}$. 
Table 2: Sensitivity analysis.

\begin{tabular}{|c|c|c|c|c|c|}
\hline \multirow[t]{2}{*}{ No } & \multirow{2}{*}{$\begin{array}{l}\text { Demand } \\
\left(\tilde{D}_{t}\right) \\
\end{array}$} & \multicolumn{2}{|c|}{$\tilde{C}_{a}=(14,15,19,21,25,26)$} & \multicolumn{2}{|c|}{$\tilde{C}_{a}=(14,15,18,22,25,26)$} \\
\hline & & $Q_{d}^{*}$ & $F\left(Q^{*}\right)$ & $Q_{d}^{*}$ & $F\left(Q^{*}\right)$ \\
\hline 1 & $\begin{array}{l}(200,300,450, \\
500,600,650)\end{array}$ & $\begin{array}{l}38.00, \quad 46.54, \\
57.00, \quad 60.09, \\
65.82,68.51)\end{array}$ & $\begin{array}{l}125.18, \\
178.93,298.52, \\
352.45,454.17, \\
490.06)\end{array}$ & $\begin{array}{ll}(38.00, & 46.54, \\
57.00, & 60.09, \\
65.82, & 68.51)\end{array}$ & $\begin{array}{l}(125.18, \\
178.93,298.52, \\
352.45,454.17, \\
490.06)\end{array}$ \\
\hline 2 & $\begin{array}{l}(225,325,475 \\
525,625,675)\end{array}$ & $\begin{array}{l}(40.31, \quad 48.44, \\
58.57, \quad 61.57, \\
67.18,69.82)\end{array}$ & $\begin{array}{l}(133.39, \\
186.66,306.65, \\
361.13,463.79, \\
499.69)\end{array}$ & $\begin{array}{l}(40.31, \quad 48.44, \\
58.57, \quad 61.57, \\
67.18,69.82)\end{array}$ & $\begin{array}{l}(133.39, \\
186.66,306.65, \\
361.13,463.79, \\
499.69)\end{array}$ \\
\hline 3 & $\begin{array}{l}(250,350,500 \\
550,650,700)\end{array}$ & $\begin{array}{l}(42.49, \quad 50.27, \\
60.09, \quad 63.02, \\
68.53,71.10)\end{array}$ & $\begin{array}{l}(141.25, \\
194.10,314.58, \\
369.62,473.21, \\
509.17)\end{array}$ & $\begin{array}{l}(42.49, \quad 50.27, \\
60.09, \quad 63.02, \\
68.53,71.10)\end{array}$ & $\begin{array}{l}(141.25, \\
194.10,314.58, \\
369.62,473.21, \\
509.17)\end{array}$ \\
\hline 4 & $\begin{array}{l}(275,375,525 \\
575,675,725)\end{array}$ & $\begin{array}{l}(44.56, \quad 52.04, \\
61.57, \quad 64.44, \\
69.82,72.36)\end{array}$ & $\begin{array}{l}(148.82, \\
201.29,322.32, \\
377.91,482.49, \\
518.52)\end{array}$ & $\begin{array}{l}(44.56, \quad 52.04, \\
61.57, \quad 64.44, \\
69.82,72.36)\end{array}$ & $\begin{array}{l}(148.82, \\
201.29,322.32, \\
377.91,482.49, \\
518.52)\end{array}$ \\
\hline 5 & $\begin{array}{l}(300,400,550, \\
600,700,750)\end{array}$ & $\begin{array}{l}(46.54, \quad 53.74, \\
63.02, \quad 55.82, \\
71.10,73.59)\end{array}$ & $\begin{array}{l}\text { (156.11, } \\
208.27,329.88, \\
386.06, \quad 491.6, \\
527.71)\end{array}$ & $\begin{array}{l}(46.54, \quad 53.74, \\
63.02, \quad 55.82, \\
71.10,73.59)\end{array}$ & $\begin{array}{l}(156.11, \\
208.27,329.88, \\
386.06, \quad 491.6, \\
527.71)\end{array}$ \\
\hline
\end{tabular}

\section{Numerical Example}

\subsection{Fuzzy Model}

Let $\quad \tilde{C}_{h}=(6,7,11,13,17,18)$,

$\tilde{C}_{a}=(14,15,19,21,25,26)$,

$\tilde{C}_{s}=(1,2,5,7,10,11)$,

$\tilde{D}_{t}=(250,350,500,550,650,700), T=6$ days then

$\tilde{Q}_{d}^{*}=(42.49,50.27,60.09,63.02,68.53,71.10)$

$F\left(\tilde{Q}^{*}\right)=(141.25,194.10,314.58,369.62,473.21,509.17)$.

From Table 2 we observed that

(i) The fuzzy economic order quantity obtained is very closer to crisp economic order quantity.

(ii) The fuzzy total cost is very closer to crisp total cost.

(iii) For different values of fuzzy ordering quantity by changing middle two 
spreads, the fuzzy economic order quantity remains fixed. The same is true for fuzzy total cost.

\section{Conclusion}

In this paper, we have studied fuzzy optimal order quantity and fuzzy optimal total cost with aid of hexagonal fuzzy number. To estimate various fuzzy optimal quantities, the demand, holding cost, ordering cost and shortage cost using hexagonal fuzzy numbers have been utilized. A new arithmetic operations of hexagonal fuzzy numbers are proposed to get the expected result. Also it is observed that the fuzzy estimates all closer to the crisp estimates of the real systems.

\section{References}

[1] Wang Chan, Backorder fuzzy inventory model under function principle, Information Science, 95 (1996), 1-2, 71-79.

[2] G. Hadley and T.M. Whitin, Analysis of Inventory Systems, Prentice-Hall, Englewood Cliffs, NJ, 1963.

[3] F. Harris, Operations and Cost, AW Shaw Co. Chicago, (1915).

[4] R. Jain, Decision making in the presence of fuzzy variables, IIIE Transactions on Systems, Man and Cybernetics, 17 (1976), 698-703.

[5] J. Kacpryzk and P. Staniewski, Long-term inventory policy-making through fuzzy-decision making models, Fuzzy Sets and Systems, 8 (1982), $117-132$.

[6] P. Parvathi and S. Gajalakshmi, An Inventory Model with Allowable Shortage Using Trapezoidal Fuzzy Numbers, International Journal of Scientific 83 Engineering Research, Volume 4, Issue 8, August-2013, ISSN 2229-5518.

[7] P. Rajarajesvari and A. SahayaSudha, A New operation on hexagonal fuzzy number, Fuzzy Logic Systems, 3 (2013) 15-26.

[8] G. Urgeletti Tinarelli, Inventory control models and problems, European Journal of Operational Research, 14 (1983), 1-12. 
[9] R. Wilson, A scientific routine for stock control, Harvard Business Review, 13 (1934), 116-128.

[10] J.S. Yao and J. Chiang, Inventory without back order with fuzzy total cost and fuzzy storing cost defuzzified by centroid and signed distance, European Journal of Operational Research, 148 (2003), 401-409.

[11] L.A. Zadeh and R.E. Bellman, Decision Making in a Fuzzy Environment, Management Science, 17 (1970), 140-16. 